\title{
Energy spectrum of Bloch electrons with two-dimensional magnetic flux modulation
}

\author{
Q. W. Shi and K. Y. Szeto \\ Department of Physics, The Hong Kong University of Science and Technology, Clear Water Bay, Kowloon, Hong Kong, China
}

(Received 6 May 1997)

\begin{abstract}
The energy spectrum of electrons on a square lattice under a uniform magnetic field $B_{0}$ and a twodimensional magnetic modulation $B_{1}$ is calculated. When the flux of $B_{0}$ is a rational multiple $p / q$ of the fluxon, we find that the Bloch band is broken into $q$ subbands for even $q$ and into $2 q$ subbands for odd $q$. Symmetry of the modified Hofstadter spectrum is discussed. [S0163-1829(97)04239-2]
\end{abstract}

The electronic structure of tight-binding electrons in two dimensions under a uniform magnetic field has been studied for many years. ${ }^{1-5}$ The spectrum shows a rich behavior such as the Hofstadter's butterfly, but so far it remains an elusive theoretical result for the experimentalists as an extremely high magnetic field in the order of $10^{9} \mathrm{G}$ is required to verify the predictions in a crystal sample. However, recent advances in submicronmeter techniques have made it possible to fabricate a lateral surface superlattice with period of the order of $100 \mathrm{~nm}$ using a two-dimensional electron gas (2DEG) in $\mathrm{GaAs} / \mathrm{Al}_{x} \mathrm{Ga}_{1-x} \mathrm{As}$ heterostructures. In these 2DEG's, one observes interesting magnetotransport phenomena even at moderate magnetic fields, leading to a renewal of interest in the subject. $^{6-12}$ Some recent highlights are the Weiss oscillations, ${ }^{6}$ which is an oscillatory magnetoresistance observed in weak magnetic fields, the transport experiments that reveal some signs of the butterfly ${ }^{13-15}$ in a twodimensional electric potential modulation system, and the symmetry breakings found in the theoretical studies of the energy spectrum of Bloch electrons under one-dimensional magnetic modulation by Gumbs et al. ${ }^{16}$ To pursue the signitures of the Hofstadter spectrum, we study the energy spectrum of Bloch electrons under two-dimensional magnetic flux modulation with a magnetic field $\vec{B}=\left[B_{0}+(-)^{m-n} B_{1}\right] \hat{z}$ going through the $(m, n)$ plaquette where $\vec{r}=m a \hat{x}+n a \hat{y}$ also labels the lower left-hand corner of the plaquette. Here $B_{0}$ is the uniform field and $B_{1}$ is the two-dimensional modulating field. This $2 \mathrm{D}$ modulating field modifies the Harper equation by the addition of a fluxdependent phase factor. In contrast to one-dimensional modulation, there is no symmetry breaking in the energy spectrum. Furthermore, we find interesting difference in the spectrum for specific values of the uniform field.

Consider a charge particle hopping on a square lattice, with hopping amplitude $t$ in the presence of an external twodimensional magnetic field $\vec{B}$. Let $|m, n\rangle$ be the Wannier state localized at site $(m, n)$. The tight-binding Hamiltonian is

$$
\begin{aligned}
\hat{H}= & -t \sum_{m, n}\left(|m, n\rangle e^{i(e a / \hbar c) A_{x}(m, n)}\langle m+1, n|+| m, n\rangle\right. \\
& \left.\times e^{i(e a / \hbar c) A_{y}(m, n)}\langle m, n+1|+\text { H.c. }\right) .
\end{aligned}
$$

The vector potential $A_{j}(m, n)(j=x, y)$ resides on the links. The total flux going through any individual plaquette is $\Phi=\sum A_{j} a=B a^{2}=a^{2} B_{0}+a^{2} B_{1}(-1)^{(m-n)}$. For convenience, we choose the gauge

$$
\begin{aligned}
& A_{x}=\frac{1}{2} a B_{0}(m-n)+(-1)^{(m-n)} \frac{1}{4} a B_{1}, \\
& A_{y}=\frac{1}{2} a B_{0}(m-n)-(-1)^{(m-n)} \frac{1}{4} a B_{1}
\end{aligned}
$$

and define the parameters $\alpha \equiv B_{0} a^{2} / \phi_{0}$ and $2 \beta \equiv \pi B_{1} a^{2} / \phi_{0}$. Here we consider only rational $\alpha=p / q$, so that flux for $B_{0}$ through a plaquette is a rational fraction of the flux quantum $\phi_{0}=h c / e$. To analyze the symmetry we introduce two translations operators $\hat{T}_{1}$ and $\hat{T}_{2}$ which commute with $\hat{H}$

$$
\hat{T}_{1}=\sum_{m, n}|m, n\rangle\langle m+1, n+1|
$$

$$
\hat{T}_{2}=\sum_{m, n}|m, n\rangle e^{-i 2 \pi(p / q)(m+n)}\langle m-1, n+1| .
$$

However, the operators $\hat{T}_{j}(j=1,2)$ do not commute with each other, as $\hat{T}_{1} \hat{T}_{2}=\hat{T}_{2} \hat{T}_{1} e^{-i 4 \pi(p / q)}$. For even $q$, we see that the operators $\hat{T}_{1}, \hat{T}_{2}^{q / 2}$, and $\hat{H}$ form a commuting set, and their eigenstates can be used to label the eigenstates of $\hat{H}$. But for odd $q$, we have to choose the eigenstates of $\hat{T}_{1}$ and $\hat{T}_{2}^{q}$ to label the eigenstates of $\hat{H}$. Physically, this even and odd difference is due to the commensurability between the Brillouin zone for the 2D periodic magnetic field and the magnetic Brillouin zone for the uniform magnetic field. Without the uniform magnetic field, the primitive cell of $2 \mathrm{D}$ periodic magnetic modulation system will consist of two plaquettes. Without the $2 \mathrm{D}$ periodic magnetic modulation, the primitive cell is $q$ times the plaquette. Thus, for even $q$, their common primitive cell is $q$ times the plaquette, while for odd $q$ their common primitive cell is $2 q$ times the plaquette. 
Next, the eigenstate $|\Psi\rangle$ of the system can be expanded in terms of a set of site states $|\Psi\rangle=\Sigma_{m, n} \Psi(m, n)|m, n\rangle$, which obeys the discrete Schröedinger equation,

$$
\begin{aligned}
-t[ & e^{i \pi(p / q)(m-n)+i(-1)^{m-n} \beta} \Psi(m+1, n) \\
& +e^{-i \pi(p / q)(m-1-n)+i(-1)^{m-n}} \beta \Psi(m-1, n) \\
& +e^{i \pi(p / q)(m-n)-i(-1)^{m-n}} \beta \Psi(m, n+1) \\
& \left.+e^{-i \pi(p / q)(m+1-n)-i(-1)^{m-n}} \beta \Psi(m+1, n)\right] \\
= & E \Psi(m, n) .
\end{aligned}
$$

Note that the coefficients in Eq. (4) only involve $m-n$. Thus we define

$$
\begin{aligned}
\Psi(m, n) & =\Psi(m-n, m+n) \\
& =e^{i v(m+n)} e^{i(\pi p / 2 q)(m-n)} g_{v}(m-n),
\end{aligned}
$$

and use it in Eq. (4) to get the modified Harper equation,

$$
\begin{aligned}
2 \cos & {\left[\pi\left(M-\frac{1}{2}\right) \alpha-(-1)^{M} \beta+v\right] g_{v}(M-1) } \\
& +2 \cos \left[\pi\left(M+\frac{1}{2}\right) \alpha+(-1)^{M} \beta+v\right] g_{v}(M+1) \\
& =\epsilon g_{v}(M)
\end{aligned}
$$

where $\epsilon=E / t$ and $v$ is the wave number along the direction which keeps $M=m-n$ constant. For rational $\alpha$ and any $\beta$, Eq. (6) is invariant under the transformation $M \rightarrow M+2 q$. However, when the periodic magnetic flux is absent $(\beta=0)$ or when $q$ is even, Eq. (6) is also invariant under the transformation $M \rightarrow M+q$. For simplicity, we use the Bloch theorem which keeps Eq. (6) invariant under the transformation $M \rightarrow M+2 q$, and introduce the following representation, $g_{v}(M)=e^{i u M} f_{v, u}(M)$ for $-(\pi / 2 q) \leqslant u \leqslant(\pi / 2 q)$, where $u$ is the wave number along the direction which keeps $m+n$ constant, and $f_{v, u}(M)=f_{v, u}(M+2 q)$ with boundary condition $f_{v+2 \pi, u+2 \pi / 2 q}(M)=f_{v, u}(M)$. Now we only need to solve the problem within the magnetic Brillouin zone $-\pi \leqslant v \leqslant \pi$ and $-(\pi / 2 q) \leqslant u \leqslant(\pi / 2 q)$. In this case, let us define $\Delta_{M}=2 \cos \left[\pi \alpha(M+1 / 2)+(-1)^{M} \beta+v\right]$. Then Eq. (6) becomes $A \vec{f}=\epsilon \vec{f}$, where $\vec{f}=\left(f_{v, u}(1), f_{v, u}(2), \ldots, f_{v, u}(2 q)\right)^{T}$ and $A$ is Hermitian,

$$
A=\left(\begin{array}{cccccccc}
0 & \Delta_{1} & 0 & 0 & \ldots & 0 & 0 & \Delta_{2 q} e^{-i 2 q u} \\
\Delta_{1} & 0 & \Delta_{2} & 0 & \ldots & 0 & 0 & 0 \\
0 & \Delta_{2} & 0 & \Delta_{3} & \ldots & 0 & 0 & 0 \\
\ldots & \ldots & \ldots & \ldots & \ldots & \ldots & \ldots & \ldots \\
0 & 0 & 0 & 0 & \ldots & \Delta_{2 q-2} & 0 & \Delta_{2 q-1} \\
\Delta_{2 q} e^{i 2 q u} & 0 & 0 & 0 & \ldots & 0 & \Delta_{2 q-1} & 0
\end{array}\right) .
$$

Consequently, there are $2 q$ real eigenvalues corresponding to the splitting of the single tight-binding Bloch band into $2 q$ subbands. Each subbands depends continuously on $v, u, \alpha$, and $\beta$.

For an infinite lattice, $A$ has several general symmetries that are independent of $\beta$ : (i) $A \rightarrow A^{\dagger}$ when $-u \rightarrow u$, the eigenvalues remain unchanged. Of course, we have the same results under the transformation $v \rightarrow-v$ for any $\beta$. This is different from the case of $1 \mathrm{D}$ magnetic modulation where no such symmetry exists, implying the symmetry breaking in 1D magnetic modulation will be absent in 2D. (ii) When $u \rightarrow u+j 2 \pi / 2 q$ or $v \rightarrow v+j 2 \pi$, for any integer $j, A$ is unchanged, which implies that all the eigenvalues have period $2 \pi / 2 q$ in the $u$ space and have period $2 \pi$ in the $v$ space. Moreover, for $v \rightarrow v+j 2 \pi p / q$, the corresponding change in Eq. (6) is $M \rightarrow M-2 j$ and the eigenvalues are unchanged. (iii) For the transformations $\alpha \rightarrow 2-\alpha$ and $-\alpha \rightarrow \alpha$, we have $M \rightarrow-M$ and the eigenvalues are unchanged.
In our calculations, we limit the values of $q$ to lie within the range $1<q \leqslant 50$ and $1 \leqslant p<2 q$. The eigenvalues of $A$ in the Eq. (7) have been found for $2 q$ values from 2 to $\overline{100}$. Values of $p$ run from 1 to $2 q-1$. Figure 1 shows plots of the scaled eigenvalues $\epsilon$ as a function of $\alpha$ for $\beta=0$ in Fig. 1(a) and $\beta=0.2 \pi$ in Fig. 1(b). For each $q$, and $1 \leqslant p<2 q$, Fig. 1(a) reproduces the Hofstadter butterfly. Note the reflection symmetry between $-\epsilon$ and $\epsilon$ in the butterfly. When 2D magnetic modulation is introduced, as shown Fig. 1(b), the fractal structure remains but is quite different from the Hofstadter's butterfly.

We illustrate the even and odd $q$ effect in two cases. For even $q(p=1, q=2)$, the spectrum is shown for $\beta=0$ in Fig. 2(a) and $\beta=0.2 \pi$ in Fig. 2(b). There are four split subbands corresponding to four eigenvalues of $A$. The symmetry between $-\epsilon$ and $\epsilon,-u$ and $u$ can be clearly seen in the region between the center and boundary of the magnetic Brillouin zone. One observes that the energy dispersion is changed 

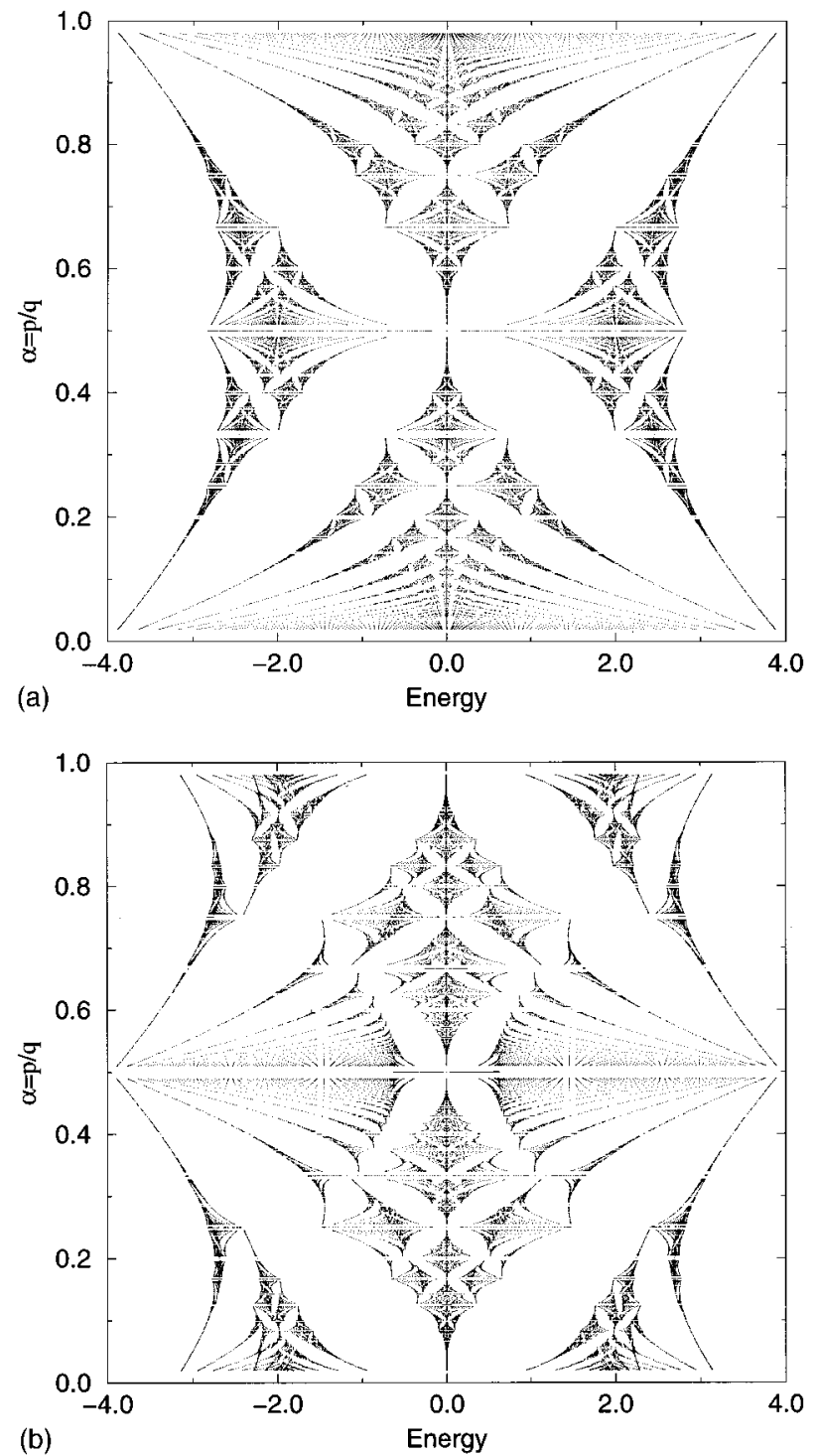

FIG. 1. Scaled eigenvalues $\epsilon=E / t$ (horizontal axis) versus $\alpha$ (vertical axis) for (a) $\beta=0$ (b) and $\beta=0.2 \pi$. Here $\alpha$ is the ratio of the uniform magnetic flux through the unit cell of area $a^{2}$ and the flux quantum. The values of $\alpha$ is $p / q$ where $1<q \leqslant 50$, and $p$ satisfies $1 \leqslant p<2 q$ for each value of $q(u=v=0)$.

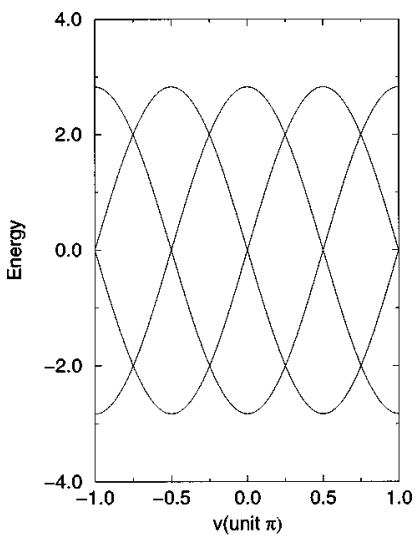

(a)

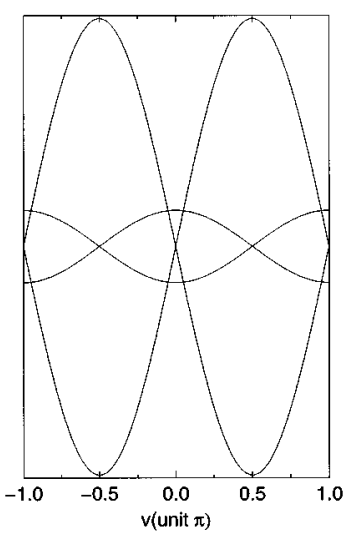

(b)
FIG. 2. Scaled energy dispersion as a function of the wave number $v$ (unit $\pi$ ) for (a) $\beta=0$ and (b) $\beta=0.2 \pi$ as $u=0$, and $\alpha=1 / 2$ with $q=2$.

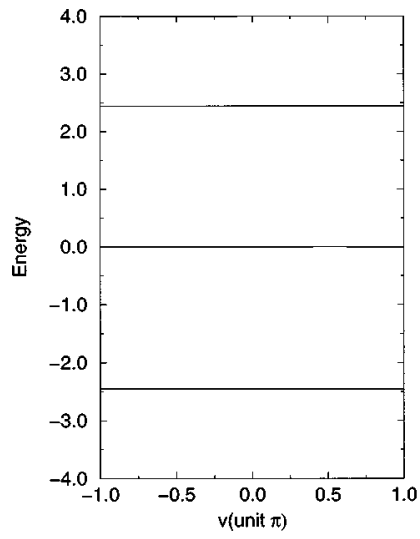

(a)

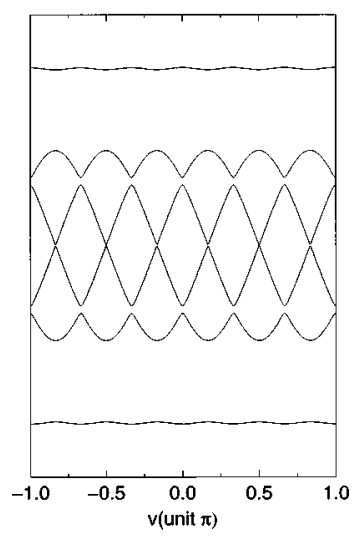

(b)
FIG. 3. Scaled energy dispersion as a function of the wave number $v$ (unit $\pi$ ) for (a) $\beta=0$ and (b) $\beta=0.2 \pi$ as $u=0$, and $\alpha=1 / 3$ with $q=3$.

under the 2D magnetic modulation. Each pair of the four subbands is connected without gaps in the Brillouin zone. This is due to our calculation in the double primitive cell for even $q$. This result agrees with our discussion using the magnetic translation operator. For odd $q(p=1, q=3)$, the energy spectrum is shown for $\beta=0$ in Fig. 3(a) and $\beta=0.2 \pi$ in Fig. $3(b)$. There are six subbands corresponding to six eigenvalues of $A$. However, without the $2 \mathrm{D}$ periodic magnetic modulation $(\beta=0)$, six subbands become three accidentally degenerate subbands since the primitive cell is $q$ times the plaquette and our numerical calculation is performed in the double primitive cell. The reflection symmetries between $-\epsilon$ and $\epsilon,-u$ and $u$ are maintained. When the $2 \mathrm{D}$ modulation is introduced, the primitive cell does change, these accidentally degenerate subbands separate into six subbands with gaps. This is quite different from the even $q$ case, as the primitive cell under the 2D magnetic flux modulation is $2 q$ times the plaquette for odd $q$.

In conclusion, we have presented numerical results for the energy subband structure of $2 \mathrm{DEG}$ in a perpendicular 2D modulated magnetic field. The symmetries between $-\epsilon$ and $\epsilon$, and $-u$ and $u$ are observed. Furthermore, we find distinct difference in the spectrum for odd and even $q$ in the uniform field parameter $\alpha=p / q$. This has many implications on transport measurements. For example, the opening of energy gaps for odd $q$ inside the Hofstadter's butterfly will greatly change the quantized Hall conductivity. An interesting application will be the preparation of a sample under 2D magnetic modulation and a uniform field changing from $\alpha=1 / 2$ to $\alpha=1 / 3$, then gaps open in the middle of subbands. This phenomenon can be observed through optical or transport experiment, as a test of our theory or as a sensitive probe for the Hofstadter butterfly. Finally, there remain many interesting problems for irrational values of $\alpha$.

\section{ACKNOWLEDGMENTS}

K.Y. Szeto acknowledges the support of the RGC Grant Nos. HKUST611/95 and HKUST685/96P. We are thankful for the discussions with X. Yan, Z. Zhang, and P. Sheng. 
${ }^{1}$ M. Ya. Azbel, Sov. Phys. JETP 19, 634 (1964).

${ }^{2}$ D. R. Hofstadter, Phys. Rev. B 14, 2239 (1976).

${ }^{3}$ F. H. Claro and G. H. Wannier, Phys. Rev. B 19, 6068 (1979).

${ }^{4}$ Y. Hatsugai and M. Kohmoto, Phys. Rev. B 42, 8282 (1990).

${ }^{5}$ J. H. Han, D. J. Thouless, H. Hiramoto, and M. Kohmoto, Phys. Rev. B 50, 11365 (1994).

${ }^{6}$ D. Weiss, et al. Europhys. Lett. 8, 179 (1989); Phys. Rev. B 39, 13020 (1989); High Magnetic Fields in Semiconductor Physics II, edited by G. Landwehr (Springer, Berlin, 1989), p. 357.

${ }^{7}$ P. Vasoilopoulos and F. M. Peeters, Phys. Rev. Lett. 63, 2120 (1989).

${ }^{8}$ R. R. Gerhardts and C. Zhang, Phys. Rev. Lett. 64, 1473 (1990).

${ }^{9}$ C. W. J Beenakker and H. van Houten, Solid State Physics: Ad- vances in Research and Applications, edited by H. Ehrenreich and D. Turnbull (Academic, New York, 1991), Vol. 44.

${ }^{10}$ X. Wu and S. E. Ulloa, Phys. Rev. B 47, 7182 (1993).

${ }^{11}$ Q. W. Shi and K. Y. Szeto, Phys. Rev. B 53, 12990 (1996).

${ }^{12}$ Q. W. Shi and K. Y. Szeto, Phys. Rev. B 55, 4558 (1997).

${ }^{13}$ D. Weiss, A. Menschig, K. von Klitzing, and G. Weimann, Surf. Sci. 263, 314 (1990).

${ }^{14}$ R. R. Gerhardts, D. Weiss, and U. Wulf, Phys. Rev. B 43, 5192 (1991).

${ }^{15}$ D. Pfannkuche and R. R. Gerhardts, Phys. Rev. B 46, 12606 (1992).

${ }^{16}$ G. Gumbs, D. Miessein, and D. H. Huang, Phys. Rev. B 52, 14 755 (1995). 